\title{
Oral myiasis in a cerebral palsy patient: A case report
}

\author{
Jain $\mathrm{S}^{1}$, Gupta $\mathrm{S}^{2}$, Jindal SK ${ }^{3}$, Singla $\mathrm{A}^{4}$ \\ ${ }^{1}$ Professor, Department of Oral and Maxillofacial Surgery, Swami Devi Dyal Dental College and Hospital, Panchkula, India. \\ ${ }^{2}$ Reader, Department of Periodontology, Bhojia Dental College \& Hospital, Vill. Bhud, Baddi, Tehsil Nalagarh, Himachal Pra- \\ desh. \\ ${ }^{3}$ Senior Lecturer, Department of Oral Medicine and Radiology, Bhojia Dental College \& Hospital, Vill. Bhud, Baddi, Tehsil \\ Nalagarh, Himachal Pradesh. \\ ${ }^{4}$ Senior Lecturer, Department of Pedodontics and Preventive Dentistry, Bhojia Dental College \& Hospital, Vill. Bhud, Baddi, \\ Tehsil Nalagarh, Himachal Pradesh.
}

Correspondence:

Dr. Sanjeev Kumar Jindal,

Senior Lecturer,

Department of Oral Medicine \& Radiology,

Bhojia Dental College \& Hospital

Vill. Bhud, Baddi, Tehsil Nalagarh,

Himachal Pradesh - 173205, INDIA

E-mail;saj2908@yahoo.com

Received: $15 / 03 / 2010$

Accepted: $12 / 04 / 2010$
Jain S, Gupta S, Jindal SK, Singla A. Oral myiasis in a cerebral palsy patient: A case report. J Clin Exp Dent. 2010;2(2):e110-2.

http://www.medicinaoral.com/odo/volumenes/v2i2/jcedv2i2p110.pdf

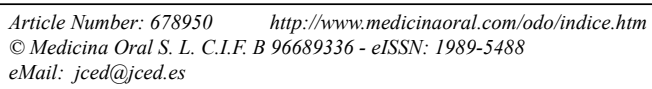

\begin{abstract}
Myiasis is a rare condition caused by the invasion of tissues by the larvae of flies, mainly from the order of Diptera. Many cases of myiasis involving various human organs have been reported. Oral Myiasis is very rare in healthy persons. It occurs mainly in the tropics and is usually associated with inadequate personal and public hygiene; sometimes accompanied by poor manual dexterity. We present a case of oral myiasis in a mentally retarded, 13year-old boy suffering with cerebral palsy. The diagnosis was based on the characteristic clinical features and the visualization of wriggling larvae. Large numbers of larvae were found in the gingival sulcus. Treatment consisted of manual removal of the larvae, one by one, with the help of turpentine oil and clinical forceps, local debridement of the labial gingival and oral hygiene practice. Healing was uneventful, with resolution of the condition.
\end{abstract}

Key words: Oral myiasis, cerebral palsy, parasitology. 


\section{Introduction}

The term Myiasis derived from the Greek word "myia" meaning fly and "iasis" meaning disease was coined by Hope in 1840 (1). Myiasis is rare and the average practitioner of pediatrics is unlikely to ever see a case. Myiasis was defined as infestation of live human and vertebrate animals with dipterous larvae which feed on the host's dead or living tissue, liquid body substances, or ingested food (2). The most common sites for myiasis are the nose, eye, lung, ear, anus, vagina and more rarely, the mouth. Incidence of oral myiasis as compared to cutaneous myiasis is less as the oral tissues are not permanently exposed to the oral environment. In orofacial myiasis, soft tissues of the oral cavity are invaded by parasitic larvae of flies (3). Lawrence (4) first described oral myiasis in 1909. Since then, it has been reported mainly in developing countries such as Asian countries and very rarely from Western countries. The incidence of oral myiasis is rare, even in developing countries.

Myiasis can be classified as primary (larvae feed on living tissues) and secondary (larvae feed on dead tissue). Depending on the condition of the involved tissue into accidental myiasis (larvae ingested along with food), semi-specific (larvae laid on necrotic tissue in wounds) and obligatory myiasis (larvae affecting undamaged skin). Further classification can be based on the site as cutaneous, external orifice, internal organs and generalized (5).

The condition can be completely benign and asymptomatic, result in mild to acute pain, or in extreme cases cause death of the patient. Predisposing factors for oral myiasis are extraction wounds, poor oral hygiene, senility, mouth breathing during sleep, suppurative lesions, necrotic tissues, diabetes and perivascular diseases mainly in the elderly, severe halitosis, alcoholism, cerebral palsy, mental retardation and hemiplegia, and factors that favor persistent non-closure of the mouth $(6,7)$.

Cases of oral myiasis have been reported to occur following dental extraction, nosocomial infection, in drug addicts, following visits to tropical countries, and in psychiatric patients.

This report describes a case of gingival myiasis in the maxillary anterior region in a child with spastic cerebral palsy.

\section{Case Report}

A 13 year old boy with spastic cerebral palsy was referred to our department with the complaint of swelling of the labial gingiva accompanied by an itching sensation and a fetid odor for about 3 days. His parents also noticed worm- like structures in the maxillary anterior area. The child presented with persistent mouth opening and very poor oral hygiene. Intraoral examination revealed live maggots in the gingival sulcus of the upper right lateral incisor to the upper left lateral incisor (Fig. 1).

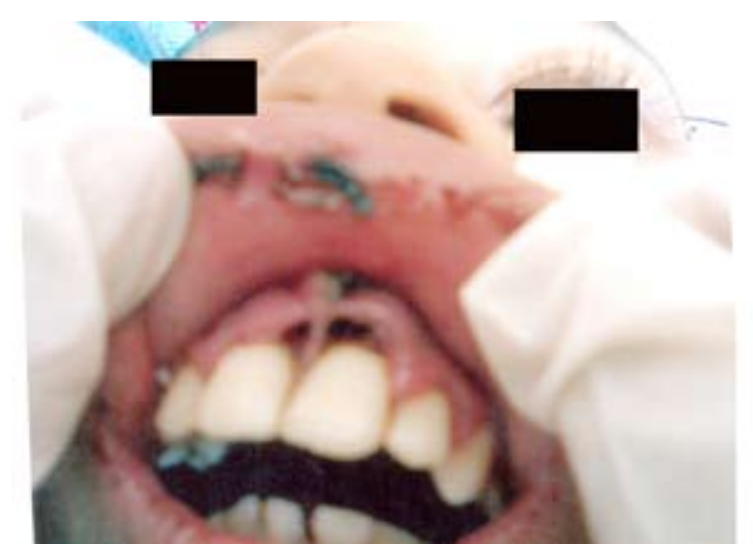

Fig. 1. Photograph showing the maggots visible in the maxillary gingival sulcus

The buccal gingiva had formed deep pockets and contained about $10-15$ visible living maggots. The first attempt was to flush the cavity with normal saline which proved ineffective. A cotton bud impregnated with turpentine was then placed at the opening of the cavity for 10 to 15 minutes. Dozens of maggots 'rushed' out from the cavity, which were then manually removed one by one, with the help of clinical forceps, and sent for entomological examination (Fig 2).

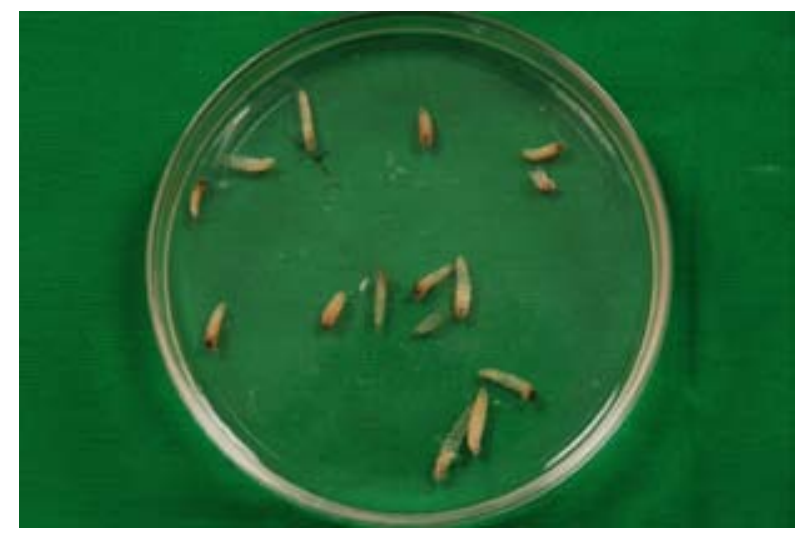

Fig. 2. Photograph depicting the live maggots that were retrieved after the application of turpentine oil

This procedure was performed once daily. By the third day, the oral cavity was free from maggots. Broad spectrum amoxicillin $250 \mathrm{mg}$ three times daily was prescribed as there were signs of infection. Oral hygiene instructions and reinforcement (to the parents and guardians) was carried out extensively and the patient was referred to Post Graduate Institute of Medical Education and Research (PGIMER), Chandigarh for further rehabilitation.

Maggots were examined by a zoologist and revealed as house fly. They belong to phylum: Arthropoda, subphylum: Mandibulata, class: Insecta, order: Diptera, genus: Musca, species: Nebulo. 


\section{Discussion}

Musca nebulo is the commonest Indian house fly. They are seen in abundance in human dwellings and are very active during summer and rainy season (8). The life cycle of a fly begins with the egg stage followed by the larva, the pupa and finally the adult fly. The conditions required for egg laying and survival of the larvae are moisture, necrotic tissue and suitable temperature.

The patient in the present case was of low socio- economic status having poor living conditions. The poor oral hygiene, lack of manual dexterity, lip incompetence, open bite and residence in a rural area were considered to be predisposing factors for larval infestation in this patient. It was predicted that the flies were attracted to the bad mouth odor due to neglected oral hygiene or fermenting food debris, making our patient a prime target for this disease. In addition, the patient was mentally retarded and dependant on his relatives for day-to-day activities which could be thought of as a contributing factor to his neglected oral hygiene.

The developmental transition via the larval stage requires an intermediate host. The periodontal pockets contributed for the mechanical support, and provided suitable substrate and temperature for the survival of the larvae. The larval stage lasts from six to eight days in which period they are parasitic to human beings. They are photophobic and therefore tend to hide themselves deep into the tissues and also to secure a suitable niche to develop into pupa $(9,10)$. The present case also showed the larvae burrowed deep inside the pockets. Proteolytic enzymes released by the surrounding bacteria decompose the tissue and the larvae feed on this rotten tissue (11). The infected tissue frequently releases foul smelling discharge.

Primary oral myiasis commonly affects the anterior part of the mouth, as in the case reported here. Oral myiasis can also be classified as:

1) Larvae living outside the body.

2) Larvae burrow into unbroken skin and develop under it.

3) Larvae live in the intestinal or urinary passages.

4) Eggs or young larvae are deposited in the wounds or natural cavities in the body.

Our case report correlates with the fourth group in the above classification.

The traditional management of myiasis is the mechanical removal of the maggots. In case of multiple larvae and in advanced stages of development and tissue destruction, local application of several substances such as oil of turpentine, larvicidal drug like Negasunt ${ }^{\circledR}(12)$, mineral oil, ether, chloroform, ethyl chloride, mercuric chloride, creosote, saline, phenol, calomel, olive oil, iodoform, can be used to ensure complete removal of all larvae $(13,14)$. Turpentine is a toxic chemical as it can induce tissue necrosis. When applied topically, it can produce epithelial hyperplasia, hyperkeratosis and ulceration. However, the damage is reversible, the hyperplasia will only persist when the stimulus is continuously applied and regresses once it is withdrawn.

Systemic treatment includes broad-spectrum antibiotics such as ampicillin and amoxicillin especially when the wound is secondarily infected. Systemic ivermectin, (semi-synthetic macrolide antibiotic) may give favorable results in more severe cases (15).

This disease can be prevented by controlling fly population and by maintaining good oral and personal hygiene. Special needs patients include patients with mental and/or physical disability. Most of these patients have difficulties in maintaining good oral hygiene due to poor manual dexterity. Parents / guardians are too busy concentrating on the patients' social or other health aspects and may not be aware of the importance of oral hygiene or may be having difficulty in gaining access to a dental clinic. It is our duty as dentists to make them aware that a special needs patient should be exposed to the dental intervention as early as possible to promote co-operation and confidence and to prevent disease.

\section{References}

1. Hope FW. On insects and their larvae occasionally found in human body. Trans R Soc Entomol. 1840; 2: 256-71.

2. Sharma J, Mamatha GP, Acharya R. Primary oral myiasis: a case report. Med Oral Patol Oral Cir Bucal. 2008;13:E714-6.

3. Aguiar AM, Enwonwu CO, Pires FR. Noma (cancrum oris) associated with oral myiasis in an adult. Oral Dis. 2003;9:158-9.

4. Lawrence SM. Dipterous larvae infection. Br Med J. 1909; 9: 88.

5. Kamboj M, Mahajan S, Boaz K. Oral myiasis misinterpreted as salivary gland adenoma. J Clin Pathol. 2007;60:848.

6. Bhatt AP, Jayakrishnan A. Oral myiasis: a case report. Int J Paediatr Dent. 2000;10:67-70.

7. Gursel M, Aldemir OS, Ozgur Z, Ataoglu T. A rare case of gingival myiasis caused by diptera (Calliphoridae). J Clin Periodontol. 2002;29:777-80.

8. Sharma J, Mamatha GP, Acharya R. Primary oral myiasis: a case report. Med Oral Patol Oral Cir Bucal. 2008;13:E714-6.

9. Bozzo L, Lima IA, de Almeida OP, Scully C. Oral myiasis caused by sarcophagidae in an extraction wound. Oral Surg Oral Med Oral Pathol. 1992;74:733-5.

10. Josephson RL, Krajden S. An unusual nosocomial infection: nasotracheal myiasis. J Otolaryngol. 1993;22:46-7.

11. Novelli MR, Haddock A, Eveson JW. Orofacial myiasis. Br J Oral Maxillofac Surg. 1993;31:36-7.

12. Felices RR, Ogbureke KU. Oral myiasis: report of case and review of management. J Oral Maxillofac Surg. 1996;54:219-20.

13. Henry J. Oral myiasis: a case study. Dent Update. 1996;23:372-3.

14. Craig GT, Franklin CD. The effect of turpentine on hamster cheek pouch mucosa: a model of epithelial hyperplasia and hyperkeratosis. J Oral Pathol. 1977;6:268-77.

15. Victoria J, Trujillo R, Barreto M. Myiasis: a successful treatment with topical ivermectin. Int J Dermatol. 1999;38:142-4. 